\title{
Automated multi-atlas segmentation of cardiac 4D flow MRI
}

Mariana Bustamante, Vikas Gupta, Daniel Forsberg, Carljohan Carlhäll, J an Engvall and Tino Ebbers

The self-archived postprint version of this journal article is available at Linköping University Institutional Repository (DiVA):

http:// urn.kb.se/ resolve?urn=urn:nbn:se:liu:diva- 150788

N.B.: When citing this work, cite the original publication.

Bustamante, M., Gupta, V., Forsberg, D., Carlhäll, C., Engvall, J., Ebbers, T., (2018), Automated multiatlas segmentation of cardiac 4D flow MRI, Medical Image Analysis, 49, 128-140.

https:// doi.org/ 10.1016/j.media.2018.08.003

Original publication available at:

https:// doi.org/ 10.1016/j.media.2018.08.003

Copyright: Elsevier

http://www.elsevier.com/

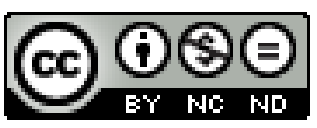




\title{
Automated multi-atlas segmentation of cardiac 4D Flow MRI
}

\author{
Mariana Bustamante ${ }^{\mathrm{a}, \mathrm{b}, *}, V_{\text {Vikas Gupta }}^{\mathrm{a}, \mathrm{b}}$, Daniel Forsberg $^{\mathrm{b}, \mathrm{c}}$, Carl-Johan Carlhäll $^{\mathrm{a}, \mathrm{d}}$, Jan Engvall ${ }^{\mathrm{a}, \mathrm{d}}$, Tino Ebbers ${ }^{\mathrm{a}, \mathrm{b}}$ \\ ${ }^{a}$ Division of Cardiovascular Medicine, Department of Medical and Health Sciences, Linköping University, Linköping, Sweden \\ ${ }^{b}$ Center for Medical Image Science and Visualization (CMIV), Linköping University, Linköping, Sweden \\ ${ }^{c}$ Sectra AB, Linköping, Sweden \\ ${ }^{d}$ Department of Clinical Physiology and Department of Medical and Health Sciences, Linköping University, Linköping, Sweden
}

Four-dimensional (4D) flow magnetic resonance imaging (4D Flow MRI) enables acquisition of time-resolved threedirectional velocity data in the entire heart and all major thoracic vessels. The segmentation of these tissues is typically performed using semi-automatic methods. Some of which primarily rely on the velocity data and result in a segmentation of the vessels only during the systolic phases. Other methods, mostly applied on the heart, rely on separately acquired balanced Steady State Free Precession (b-SSFP) MR images, after which the segmentations are superimposed on the $4 \mathrm{D}$ Flow MRI. While b-SSFP images typically cover the whole cardiac cycle and have good contrast, they suffer from a number of problems, such as large slice thickness, limited coverage of the cardiac anatomy, and being prone to displacement errors caused by respiratory motion. To address these limitations we propose a multi-atlas segmentation method, which relies only on 4D Flow MRI data, to automatically generate four-dimensional segmentations that include the entire thoracic cardiovascular system present in these datasets. The approach was evaluated on 4D Flow MR datasets from a cohort of 27 healthy volunteers and 83 patients with mildly impaired systolic left-ventricular function. Comparison of manual and automatic segmentations of the cardiac chambers at end-systolic and end-diastolic timeframes showed agreements comparable to those previously reported for automatic segmentation methods of b-SSFP MR images. Furthermore, automatic segmentation of the entire thoracic cardiovascular system improves visualization of $4 \mathrm{D}$ Flow MRI and facilitates computation of hemodynamic parameters.

Keywords: 4D Flow MRI, Multi-atlas segmentation, Cardiac segmentation

\section{Introduction}

Magnetic Resonance Imaging (MRI) enables fast and accurate generation of morphological and functional images of the cardiovascular system, and is routinely performed to diagnose and assess the function of the heart and great vessels. In order to extract relevant information from these images in the heart, one of the most important and challenging pre-processing steps is the segmentation of clinically useful cardiac regions, usually relying on manual delineation of the contours by an expert observer. A number of disadvantages can be associated with manual segmentation, such as being time-consuming, dependent on the observer's expertise, and requiring considerable efforts in order to establish standardized analysis procedures (Schulz-Menger et al., 2013, Suinesiaputra et al., 2014; Kawel-Boehm et al. 2015).

With the aim of addressing these issues, a large number of automatic and semi-automatic segmentation methods have been proposed and evaluated using cardiac MRI (Petitjean and Dacher, 2011; Bai et al., 2013, Queiros et al. 2014). These techniques usually focus on short-axis (SA)

\footnotetext{
*Corresponding author

Email address: mariana.bustamante@liu.se (Mariana Bustamante)
}

cine (time-resolved) cardiac MRI acquired using balanced Steady State Free Precession (b-SSFP) sequences, the reason being the high signal-to-noise ratio and good contrast between blood and myocardium that these images provide (Bieri and Scheffler, 2013).

Four-dimensional (4D) Flow MRI is a three-dimensional (3D) cine (time-resolved) phase-contrast MRI with threedirectional velocity encoding. Typically, 4D Flow MRI acquisitions include the entire heart and the major thoracic vessels (aorta, pulmonary arteries, caval veins, and pulmonary veins) in one volume, with isotropic or nearisotropic voxels (Markl et al., 2011). Unfortunately, the contrast between the blood pool and myocardium is extremely low in 4D Flow MRI data. The assessment of 4D Flow MRI cardiac data has, therefore, mainly been performed using segmentations created on b-SSFP SA MR images (Eriksson et al., 2010; Kanski et al., 2015; Arvidsson et al., 2016, Fredriksson et al., 2016). This can be detrimental to the flow analysis in a number of ways: (a) due to time constraints, b-SSFP SA MR images are typically limited to the left and right ventricles of the heart, (b) the entire atria are usually only included in a few longaxis (LA) two-dimensional slices, (c) b-SSFP MR images are typically acquired as two-dimensional(2D) slices, with a relatively large thickness of 6-10 mm, and (d) b-SSFP 
MR images are susceptible to misalignments caused by breathing-related motion, since the stack of images is obtained over the course of several breath-holds (Scott et al. 2009).

Automatic segmentation of 4D Flow MRI data would allow for fast, consistent, and reliable hemodynamic assessment of the entire thoracic cardiovascular system, both for research and clinical applications. Atlas-guided segmentation uses the information contained in an atlas in conjunction with image registration to generate a segmentation (Rohlfing et al., 2005). In the context of biomedical image analysis, an atlas is an image that incorporates locations and shapes of anatomical structures and the spatial relationships between them. Along the same line, multi-atlas segmentation is an extension of this technique based on the idea that using multiple atlases, as opposed to a single average or model atlas, better captures the inter-subject variability of the anatomy to be segmented (Rohlfing et al. 2004 Doan et al., 2010). This technique has been evaluated on several types of biomedical images, resulting in segmentations that are comparable or superior to other automatic techniques (Iglesias and Sabuncu, 2015).

Previously, we have reported an automatic atlas-based approach to segment the large vessels in 4D Flow MRI data (Bustamante et al., 2015). The current study improves upon this technique by incorporating multiple atlases and including a newly developed technique for the generation of angiographic data to also obtain segmentations of the cardiac chambers. Due to the low contrast between blood and myocardium present in 4D Flow MR images and the considerable variability of cardiac anatomy between patients, generating a segmentation of the heart is far from trivial. This study aims to develop and evaluate a multi-atlas segmentation method to automatically generate four-dimensional segmentations from 4D Flow MRI data including the entire thoracic cardiovascular system and, thereby, allowing blood-flow analysis within the different cardiac chambers and the great thoracic vessels.

\section{Methods}

\subsection{Multi-atlas segmentation}

Multi-atlas segmentation is a technique used to infer a label for each voxel of a target image based on the information provided by a number of images known as atlases (Rohlfing et al., 2004, Klein et al., 2005, Heckemann et al. 2006). In this context, an atlas is an image that has been labeled previously. Using registration, a transformation between the target and each atlas is estimated in order to propagate each label to the target's image space. Subsequently, the labels derived from each atlas are combined into a single final segmentation.

For this study, a set of eight atlases was created by manually segmenting specific regions of the 4D Flow MRI data with the open-source tool ITK-snap (Yushkevich et al. 2006). The atlases were initially delineated by an image analyst with four years of experience in cardiovascular
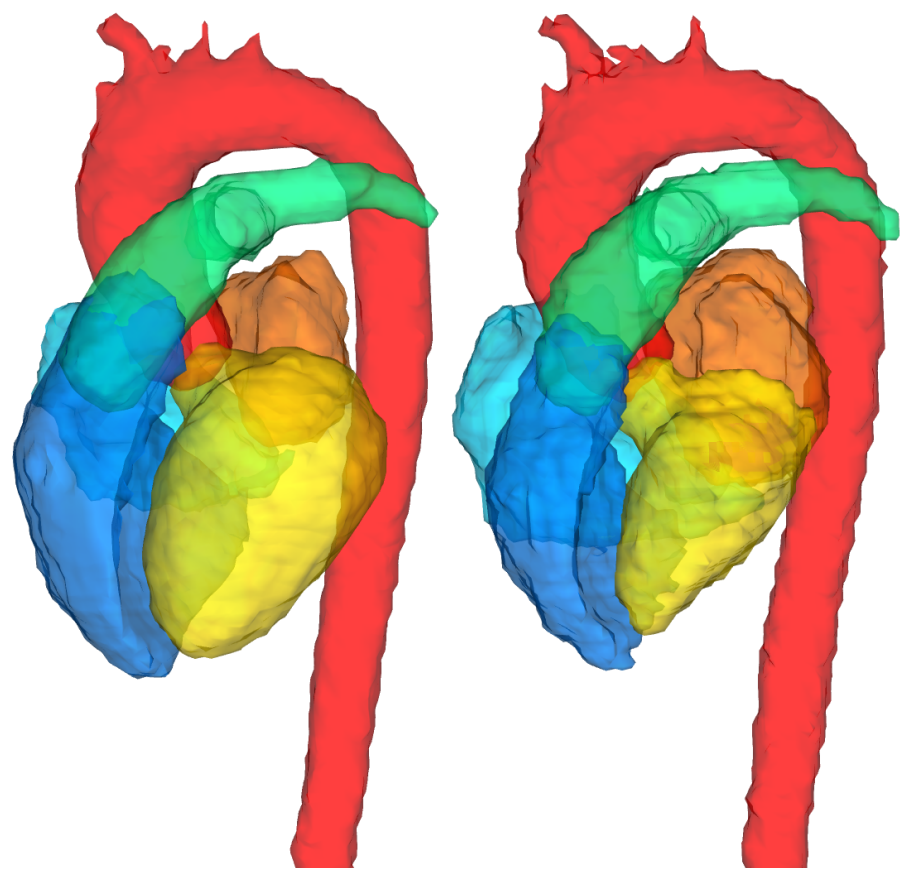

Figure 1: An atlas with manually delineated labels in different colors at end-diastole (left) and end-systole (right). The regions included are: left ventricle (yellow), left atrium (orange), right ventricle (dark blue), right atrium (light blue), aorta (red), pulmonary artery (green).

imaging, and later reviewed and corrected by a clinician with fifteen years of experience in cardiovascular imaging. Each atlas included segmentations of the left ventricle, left atrium, right ventricle, right atrium, aorta, and pulmonary artery, at both end-systolic and end-diastolic timeframes. These timeframes were selected in order to ensure that the most extreme changes in the cardiac volumes were correctly depicted. The segmentations of the left and right ventricles in the atlases were based on segmentations initially performed on b-SSFP SA MR images. Special care was taken to choose datasets where the SA stack was not severely misaligned, and corrections were made to ensure that this would not affect the final atlases. Since all four heart chambers were included in the atlases, the valve annulus planes were indirectly tracked by the separation of the atria and ventricles in these atlases. Figure 1 shows an atlas with each region highlighted in a different color.

Since each atlas provides a possible segmentation for each of the regions of interest, the final step of a multi-atlas segmentation method is to combine or fuse these labels into one final segmentation. This step was implemented using the simultaneous truth and performance level estimation (STAPLE) algorithm (Warfield et al., 2004), further described in section 2.5 .

In this way, multi-atlas segmentation was used to generate end-diastolic and end-systolic segmentations of all the regions present in the atlases. Subsequently, these segmentations were propagated to each of the remaining timeframes included in the 4D MR images using a temporal registration approach described in section 2.6. A flowchart 


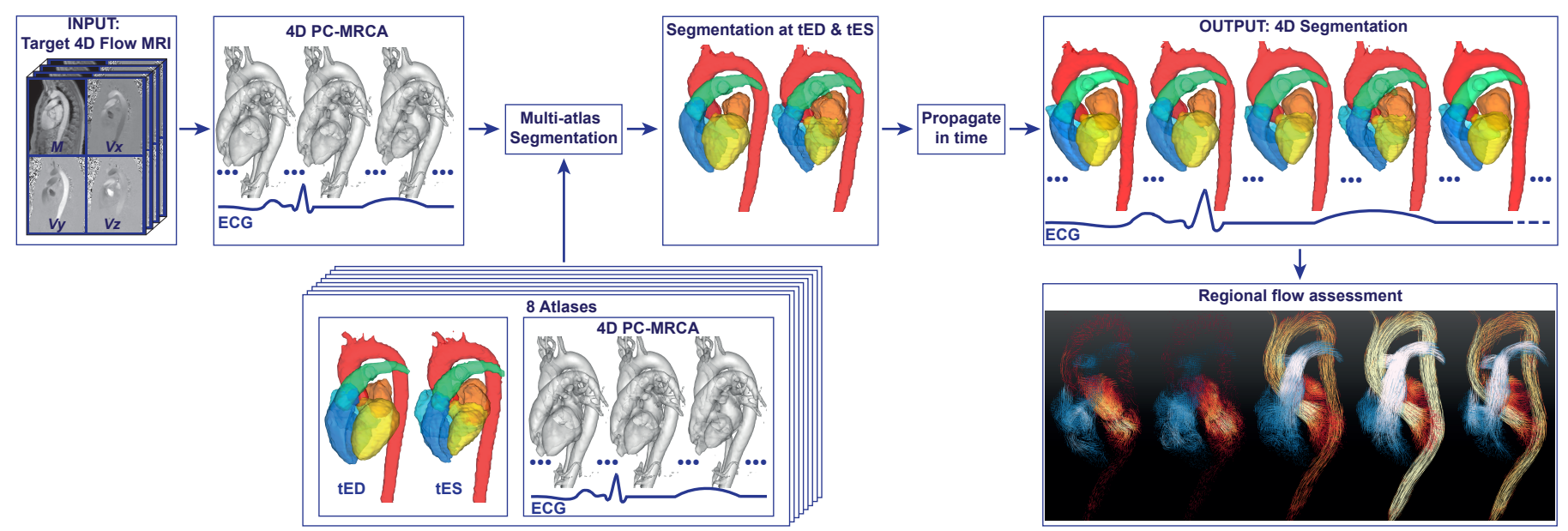

Figure 2: Flowchart of the proposed technique

of the proposed method is provided in Fig. 2 .

\subsection{The $4 D$ Flow MRI dataset}

A 4D Flow MRI dataset is composed of four volumes: A magnitude volume, $M$, depicting the anatomy of the region, and a velocity volume for each direction in the three-dimensional space: $V_{x}, V_{y}$, and $V_{z}$. These volumes are four-dimensional as each includes three dimensions of space and a dimension of time along the cardiac cycle.

\subsection{Phase-Contrast Magnetic Resonance CardioAngiogra- phy}

A 4D Phase-Contrast Magnetic Resonance CardioAngiography (4D PC-MRCA) is a time-resolved angiographic image created with the aim of improving the visualization of $4 \mathrm{D}$ Flow MRI data throughout the entire cardiac cycle.

Given a 4D Flow MRI dataset composed of a series of four-dimensional volumes including magnitude information $(M(t))$, as well as three-directional velocity information $\left(V_{x}(t), V_{y}(t), V_{z}(t)\right)$ for each timeframe $t$ in the cardiac cycle. The process of generating a $4 \mathrm{D}$ PC-MRCA can be divided into the following steps:

1. Generation of PC-MRA data from each timeframe $t$ of the $4 \mathrm{D}$ Flow MRI:

$$
\operatorname{PC}-\operatorname{MRA}(t)=M(t) *\left(V_{x}^{2}(t)+V_{y}^{2}(t)+V_{z}^{2}(t)\right)^{\gamma}
$$

where $\gamma=0.2$ improves the visibility of areas of low velocities, such as those inside the heart chambers.

2. Align all the timeframes available in the $4 \mathrm{D}$ Flow MRI to one timeframe $\hat{t}$ of the magnitude volume $M$ included in the $4 \mathrm{D}$ Flow dataset by applying nonrigid registration between each timeframe $\{M(t) \mid \forall t\}$ and $M(\hat{t})$. This results in a set of $N-1$ transformations $B=\left\{B_{1}, B_{2}, \ldots, B_{N-1}\right\}$ for $N$ timeframes in the $4 \mathrm{D}$ Flow dataset.
3. Apply the corresponding deformation field $B_{t}$ to each timeframe of the PC-MRA image created in step 1. This results in a set of images with intensities that depend on the blood flow patterns at each timeframe $t$, but with the shape and morphology expected in the chosen timeframe $\hat{t}$.

4. Generate a maximum intensity projection over time from the set of images obtained in step 3, resulting in a 3D PC-MRCA at timeframe $\hat{t}$.

5. Create a new set of non-rigid transformations $F=$ $\left\{F_{1}, F_{2}, \ldots, F_{N-1}\right\}$ by applying non-rigid registration between $M(\hat{t})$ and all timeframes $\{M(t) \mid \forall t\}$. Apply the transformations to the 3D PC-MRCA in order to obtain a time-resolved (four-dimensional) PC-MRCA.

The resulting 4D PC-MRCA image includes the whole cardiovascular system in any of the available timeframes of the 4D Flow MRI; see Fig. 3 for a representative example of a 4D PC-MRCA. Further details about the generation of 4D PC-MRCAs and their utility can be found in (Bustamante et al. 2017).

\subsection{Registration of $4 D$ Flow MRI datasets}

In order to successfully apply atlas-based segmentation on 4D Flow MRI, we need to be able to compare and register two different $4 \mathrm{D}$ Flow MRI datasets to each-other. The 4D PC-MRCA data, described in section 2.3, were used as the link between distinct datasets.

4D PC-MRCAs were generated for all the atlases and the target dataset. Two timeframes of the cardiac cycle, end-systole $\left(\hat{t}_{E S}\right)$ and end-diastole $\left(\hat{t}_{E D}\right)$, were selected in order to transfer the labels initially belonging to the atlases to the target image. Registrations between the PC-MRCAs of the atlases and the target image were performed at $\hat{t}_{E S}$ and $\hat{t}_{E D}$; this consisted of an initial affine registration step, 

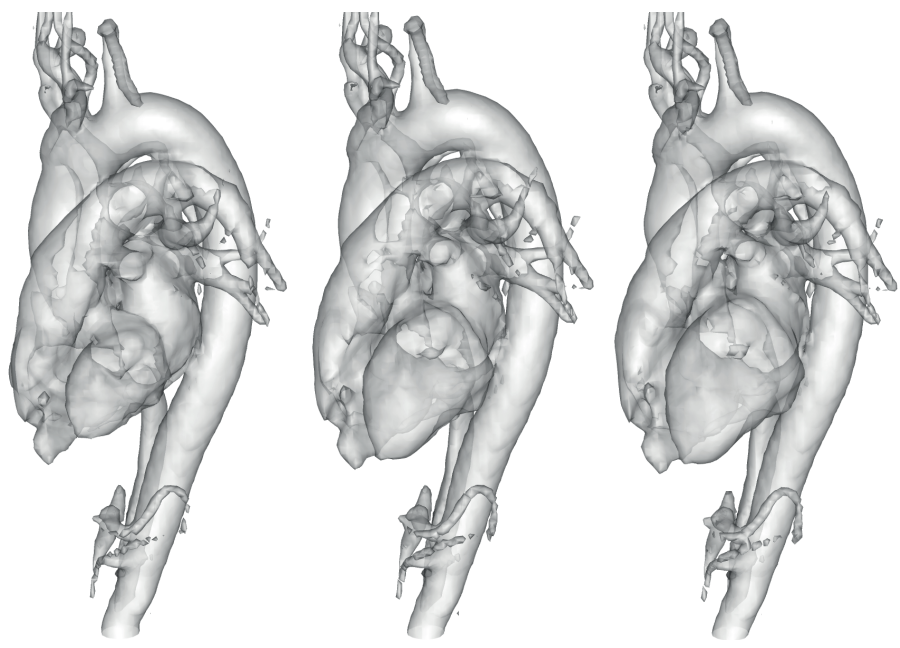

Figure 3: Isosurface visualization of a 4D Phase-Contrast Magnetic Resonance CardioAngiography (4D PC-MRCA) at three timeframes of the cardiac cycle: end-systole, mid-diastole, and end-diastole.

followed by non-rigid registration. The non-rigid registration was based on Morphon (Knutsson and Andersson, 2005), as implemented in a general non-parametric framework for image registration (Forsberg, 2013), and also used and evaluated for a number of atlas-based segmentation tasks (Forsberg et al., 2014, Forsberg, 2015). The registration method uses local phase differences computed between the moving and the target image $\left(I_{m}\right.$ and $I_{t}$, respectively) to estimate a displacement field, $u$, which subsequently can be applied to the moving image $\left(I_{m}(x+u(x))=I_{u}(x)\right)$ to align it with the target image $I_{t}$. Note that the employed local phase does not correspond to the phase contrast of the acquired image data, but refers to the phase describing local structural changes as can often be observed by a human (Fleet and Jepson, 1990). The displacement field is estimated iteratively using multiple scales that progress from coarser to finer until the deformed image is as close as possible to the target, $\left(I_{u}(x)=I_{t}(x)\right)$. The update field, $\delta u$, estimated at each step was accumulated into the displacement field $u$ using diffeomorphic field accumulation (Vercauteren et al., 2009). Additionally, fluid and elastic regularization were used in order to ensure the generation of smooth, physically plausible displacement fields. Fluid regularization acts upon the estimated updated field, $\delta u$, at each iteration, whereas elastic regularization acts on the accumulated displacement field, $u$ (Forsberg, 2013). These regularizers are defined as $L_{2}$-norms of derivatives of the displacement field $u$ :

$$
R(u)=\frac{1}{2} \int_{\Omega}\|\mathcal{B}\|^{2} d x
$$

where $\mathcal{B}$ is a differential operator integrated over the continuous domain $\Omega$.

The fluid regularization (Christensen et al., 1996) is defined as:

$$
R(v)=\frac{1}{2} \int_{\Omega} \mu\langle\nabla v, \nabla v\rangle+(\lambda+\mu)(\nabla \cdot v)^{2} d x
$$

where $v$ is a velocity field defined as $v=\partial_{t} u+v^{T} \nabla u$.

The elastic regularization (Bajcsy and Broit, 1982) is defined as:

$$
R(u)=\frac{1}{2} \int_{\Omega} \mu\langle\nabla u, \nabla u\rangle+(\lambda+\mu)(\nabla \cdot u)^{2} d x
$$

In a general framework for non-parametric image registration using a decoupling between the distance and regularization terms, the use of fluid and elastic regularization typically breaks down to applying a Gaussian smoothing kernel to the update field and accumulated displacement field respectively in each iteration.

For the registration of $4 \mathrm{D}$ PC-MRCA images, the registration parameters used were as follows:

- Number of scales: 3, corresponding to the original scale and to a downsampling of a factor of two and four.

- Number of iterations per scale: 5 .

- Gaussian kernel width $(\sigma)$ of 1.5 voxels for fluid regularization.

- Gaussian kernel width $(\sigma)$ of 1.0 voxel for elastic regularization.

\subsection{Label fusion using STAPLE}

The step of combining the registered atlas labels into one final label, usually called label fusion, was implemented using the STAPLE algorithm (Warfield et al., 2004). STAPLE uses expectation-maximization (EM) (Dempster et al. 1977) to calculate a probabilistic estimate of the true segmentation based on the group of segmentations provided by the previously deformed atlases. EM is a widely applicable approach for computing maximum likelihood estimates from incomplete data.

Expectation-maximization strategies have been used previously for label fusion in multi-atlas segmentation producing better results than simple label averaging (Rohlfing et al. 2003), in cardiac segmentation using a probabilistic atlas (Lorenzo-Valdés et al., 2004), and STAPLE in particular has been used for consensus ground truth calculation from automated and semi-automated cardiac MR segmentation methods (Suinesiaputra et al. 2014, 2015).

\subsection{Creation of a time-resolved segmentation}

While the entire cardiac cycle is included in the 4D Flow MRI datasets, the segmentations generated in the previous steps correspond only to the end-systolic and end-diastolic timeframes $\left(\hat{t}_{E S}\right.$ and $\left.\hat{t}_{E D}\right)$. The next step is, therefore, to obtain segmentations for each of the remaining timeframes. This was accomplished using the same phase-based nonrigid image registration method described previously. Similar approaches have been used in previous studies in order to adapt to the heart motion (Jolly et al., 2012, Lantz et al. 2016). Additionally, in order to ensure that the deformation fields follow the cardiac motion adequately, both the moving and target images were transformed into a local structure tensor representation of themselves (Knutsson, 
1989) - a technique that has been used previously for multimodal registration (Eklund et al., 2011, Zhang et al., 2015), further described in Appendix A. During this step, the registration parameters used were as follows:

- Number of scales: 3, corresponding to the original scale and to a resampling of a factor of two and four.

- Number of iterations per scale: 3 .

- Gaussian kernel width $(\sigma)$ of 1.5 voxels for fluid regularization.

- Gaussian kernel width $(\sigma)$ of 1.0 voxel for elastic regularization.

Registrations were executed between the different timeframes of the magnitude data $M$ of the target 4D Flow MRI dataset, which prevents the resulting deformations from being overly dependent on the cardiac motion of the atlases. The volumes $M\left(\hat{t}_{E S}\right)$ and $M\left(\hat{t}_{E D}\right)$ of the target dataset were each non-rigidly registered to the $N$ available timeframes. This resulted in two sets of displacement fields: $U_{E S}=\left\{u_{\hat{t} 1}, u_{\hat{t} 2}, \ldots, u_{\hat{t} N} \mid \hat{t}=\hat{t}_{E S}\right\}$, and $U_{E D}=\left\{u_{\hat{t} 1}, u_{\hat{t} 2}, \ldots, u_{\hat{t} N} \mid \hat{t}=\hat{t}_{E D}\right\}$, where $u_{\hat{t} i}$ is the displacement field resulting from the registration between timeframes $\hat{t}$ and $i$. These displacements were subsequently applied to the segmentations already fitted to the target dataset. This resulted in two possible volume masks, $S_{E S}$ and $S_{E D}$, depending on which timeframe was used as basis for the segmentation: end-systole, or end-diastole. The final segmentation, $S$, was obtained as a weighted sum of these masks performed at each timeframe, as shown in equation (5):

$$
S(t)=w S_{E S}(t)+(1-w) S_{E D}(t)
$$

where $w$ is equal to one at timeframes $\hat{t}_{E S}, \hat{t}_{E S}+1$, and $\hat{t}_{E S}-1$, and decreases gradually for each timeframe farther from $\hat{t}_{E S}$. The influence of $S_{E S}$ was limited to 15 timeframes; consequently, $w$ reaches a value of zero at timeframes $\hat{t}_{E S} \pm 16$. Note that the available timeframes in the data are treated as a loop that starts over once the final timeframe has been reached. For the purpose of volume calculation, a threshold of 0.8 was set to generate a binary mask from the resulting weighted sum.

It should be pointed out that all the registrations required for this step had already been obtained during the generation of the PC-MRCAs. Thus, it was not necessary to execute them again.

\section{Experimental setup}

\subsection{Study population}

The developed segmentation method was evaluated on a group of 110 subjects including 27 healthy volunteers (64 \pm 4 years of age, 5 males) with no history of prior or current cardiovascular disease or cardiac medication; and 83 patients ( $67 \pm 7$ years of age, 54 males) with suspected or diagnosed chronic ischemic heart disease based on positive myocardial scintigraphy or electrocardiography (ECG) exercise testing, and/or a history of typical or atypical angina, together with the fulfillment of the high risk score of cardiovascular disease of the European Society of Cardiology. Exclusion criteria were: presence of an intracardiac shunt, more than mild valvular disease, and ejection fraction (EF) under $55 \%$. The group composed of 83 patients consists of datasets previously included in the study DOPPLER-CIP (Rademakers et al. , 2013).

From this cohort, a set of eight datasets (six healthy volunteers and two patients with normal left ventricular function) were chosen as atlases based on good image quality.

The research was performed in line with the declaration of Helsinki and was approved by the regional ethics board. All subjects gave written informed consent.

\subsection{Acquisition settings}

The MRI examinations were performed on a clinical 3T Philips Ingenia scanner (Philips Healthcare, Best, the Netherlands). All subjects were injected with a Gadolinium contrast agent (Magnevist, Bayer Schering Pharma $\mathrm{AG}$ ) prior to the acquisition for a late-enhancement study.

4D Flow MRI examinations were performed during free-breathing, using a navigator gated gradient-echo pulse sequence with interleaved three-directional flow-encoding and retrospective vector cardiogram controlled cardiac gating. Scan parameters included: Candy cane view adjusted to cover both ventricles, velocity encoding (VENC) 120$150 \mathrm{~cm} / \mathrm{s}$, flip angle $10^{\circ}$, echo time 2.5-2.6 ms, repetition time 4.2-4.4 ms, parallel imaging (SENSE) speed up factor 3 (AP direction), k-space segmentation factor 3 , acquired temporal resolution of 33.6-52.8 ms, spatial resolution $2.7 \times 2.7 \times 2.8 \mathrm{~mm}^{3}$, and elliptical k-space acquisition. The typical scan time was 10-15 minutes including the navigator gating. The 4D Flow MR images were corrected for concomitant gradient fields on the MRI scanner. Offline processing corrected for phase wraps using a temporal phase unwrapping method (Xiang, 1995), and background phase errors were corrected using a weighted $2^{\text {nd }}$ order polynomial fit to the static tissue (Ebbers et al., 2008).

Cine MR images were acquired using a b-SSFP protocol at end-respiratory breath holds, resulting in 30 timeframes over the cardiac cycle. A short-axis stack with resolution of $1 \times 1 \mathrm{~mm}$, and slice thickness of $8 \mathrm{~mm}$ was acquired. Two-, three-, and four-chamber vertical long-axis views were also obtained with resolution of $1 \times 1 \mathrm{~mm}$.

\subsection{Cardiac volumetric parameters}

Quantification of volumetric parameters is quite common during the assessment of cardiac function. The following group of clinical parameters were also used in this study in order to evaluate the segmentation results:

- End-diastolic volume (EDV): Volume of blood in a cardiac chamber at end diastole. 
- End-systolic volume (ESV): Volume of blood in a cardiac chamber at the end of the systolic contraction.

- Stroke Volume (SV): Volume of blood pumped from a cardiac ventricle in one heart beat. It is calculated as: $S V=E D V-E S V$.

- Systemic flow volume (Qs): Measure of the volume of blood traveling from the left side of the heart to the body in a heartbeat. Calculated at a plane located in the proximal ascending aorta as:

$$
Q=\int_{t} \dot{V} d t
$$

where $t$ is the duration of the cardiac cycle, and $\dot{V}$ is the volumetric flow rate per unit time. $\dot{V}$ can be calculated as: $\dot{V}=\boldsymbol{v} \cdot \boldsymbol{A}$, where $\boldsymbol{v}$ is the flow velocity and $\boldsymbol{A}$ is the cross-sectional vector area.

- Pulmonary flow volume (Qp): Measure of the volume of blood traveling from the right side of the heart to the lungs for oxygenation in one heartbeat. Calculated in the same way as Qs, at a plane located in the pulmonary trunk.

\subsection{Evaluation criteria}

Validation of the left ventricular segmentations was done through comparison with manual segmentation of this chamber performed by experts on b-SSFP SA MR images at end-systole and end-diastole. In the same manner, the segmentations were also evaluated at a mid-systolic timeframe for a subset of 30 randomly selected datasets. All the manual segmentations were performed using the visualization software Segment v1.9 (Medviso, Lund, Sweden) (Heiberg et al., 2010).

The manual segmentations available for this study followed the procedure outlined in (Hudsmith et al., 2005), according to which a basal slice is only included in the segmentation when at least fifty percent of the blood volume is surrounded by myocardium. To evaluate the accuracy of the segmentations, two sets of left ventricular atlases were created: One matching the conditions of the manual segmentations at the cardiac base, and another including the entire left ventricular outflow tract. The volume comparisons between the automatic and manual segmentations were done with the first set of atlases, while the segmentations used to compare stroke volumes correspond to the second set.

In a similar manner, the left atrial segmentations were compared to manual segmentations performed by experts at end-systole and end-diastole. In this case however, due to the lack of coverage of the atrial regions available in the short-axis images, the left atrial volumes were approximated by volume integration from the available long-axis images included in the study (Lang et al. 2017). See Appendix B for more details about this calculation.
The right ventricle segmentations were evaluated through comparison of the stroke volumes obtained for this ventricle and the left ventricle, which should remain similar in cardiac patients without shunts or significant valvular regurgitation. Assessment of the aortic and pulmonary artery segmentations was done through comparison of the pulmonary and systemic volume flows (Qp, Qs, respectively) obtained on planes located on the ascending aorta and the pulmonary artery using a previously described method (Bustamante et al. 2015). This method also allowed further evaluation of the ventricular segmentations by comparing the ventricular stroke volumes to the Qp and Qs volume flows obtained in the vessels.

For each region, Bland-Altman analysis was used to assess the agreement between the manually segmented volumes and those generated automatically with the proposed method. Previously published state-of-the-art automatic segmentation methods have reported limits of agreement around $30 \mathrm{ml}$ when applied to cardiac MRI (Jolly et al. 2012, Bai et al., 2013, Queiros et al., 2014, Shahzad et al. 2017).

Furthermore, the manual and automatic segmentations of the left ventricle were compared using the Dice similarity coefficient in order to measure the spatial overlap between the segmentations. A previously evaluated method for inter-slice misalignment correction was applied to the bSSFP SA MR images in order to diminish the effect of patient motion and respiratory drift in these images (Gupta et al. 2017).

\section{Results}

Four-dimensional segmentations of the cardiac chambers and great thoracic vessels were generated for all the datasets using the proposed method. Fig. 4 shows two representative examples of the obtained segmentations. The left ventricular segmentation results of the same datasets are depicted in Fig. 5 compared to the manual segmentations. Note that the automatic segmentations in this image contain the left ventricular outflow tract, while the manual segmentations did not include this region. Only two timeframes are depicted due to lack of space, although all the segmentations generated are four-dimensional and include 40 timeframes.

Figure 6 shows Bland-Altman plots comparing the left ventricular volumes obtained through manual segmentation of b-SSFP SA MR images with the volumes obtained automatically using the described technique. Mean differences obtained were $-2.08 \pm 15.59 \mathrm{ml},-0.28 \pm 9.38 \mathrm{ml}$, for the left ventricular EDV and ESV, respectively. For a subset of 30 randomly selected datasets, the mean differences of left ventricular volumes at mid-systole were found to be $1.90 \pm 7.30 \mathrm{ml}$ (see Fig. 7). Similar comparisons were made for the left atrial volumes, which resulted in mean differences of $2.02 \pm 9.89 \mathrm{ml}, 6.87 \pm 14.22 \mathrm{ml}$ for the EDV, and ESV, respectively. Bland-Altman plots corresponding to the left atrial volumes can be seen in Fig. 8. 

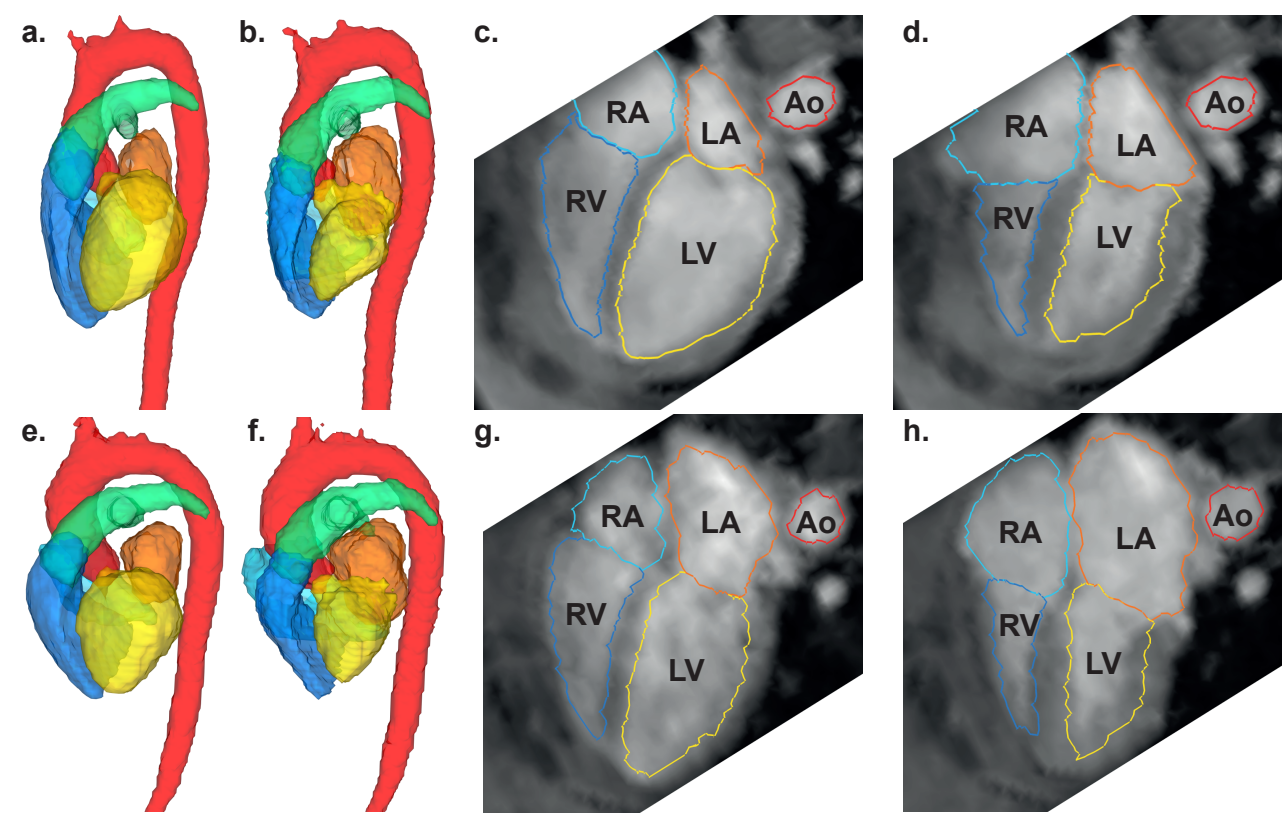

Figure 4: Result obtained for two datasets at end-diastole $(\mathbf{a}, \mathbf{e}, \mathbf{c}, \mathbf{g})$ and end-systole $(\mathbf{b}, \mathbf{f}, \mathbf{d}, \mathbf{h})$. Visualized as an isosurface rendering (a, $\mathbf{b}$, $\mathbf{e}, \mathbf{f})$, and superimposed over a four-chamber image of the heart $(\mathbf{c}, \mathbf{d}, \mathbf{g}, \mathbf{h})$. The regions included are: left ventricle (LV, yellow), left atrium (LA, orange), right ventricle (RV, dark blue), right atrium (RA, light blue), aorta (Ao, red), pulmonary artery (green).
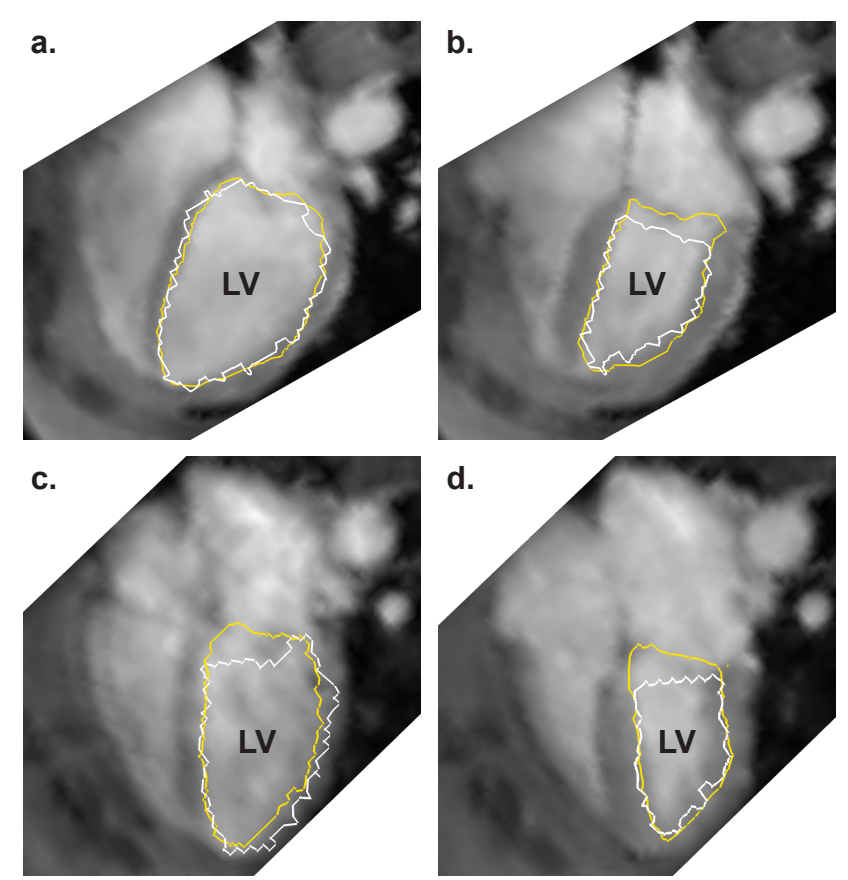

Figure 5: Left ventricular results obtained for two datasets at enddiastole $(\mathbf{a}, \mathbf{c})$ and end-systole $(\mathbf{b}, \mathbf{d})$. In order to compare the two labels, the manual segmentations performed on the b-SSFP MR were resampled and interpolated to the $4 \mathrm{D}$ Flow MRI space. The manual segmentation (white) was superimposed over the automatic segmentation result (yellow) on a four-chamber image of the heart.
The stroke volumes of the left and right ventricle obtained with the proposed method were also compared. This comparison can be seen in Fig. 9 and resulted in mean differences of $0.89 \pm 13.71 \mathrm{ml}$.

Figure 10 shows a comparison of the systemic (Qs) and pulmonary $(\mathrm{Qp})$ volume flows obtained in the aorta and pulmonary artery compared to each other and to the stroke volumes of the ventricles. Following the flow of blood through the cardiovascular system, the left ventricular stroke volumes were compared to Qs, while the stroke volumes of the right ventricle were compared to Qp. The mean differences found were $3.32 \pm 14.91 \mathrm{ml}$, and $0.97 \pm 13.45 \mathrm{ml}$ for the systemic and pulmonary flow, respectively, when compared to the ventricular stroke volumes. The mean difference between the $\mathrm{Qp}$ and $\mathrm{Qs}$ values was $3.24 \pm 9.6 \mathrm{ml}$.

For the left ventricle and left atrium, the percentage of the chamber volume that has been underestimated or overestimated by the method when compared to the manual segmentation is shown in Fig. 11 .

Figure 12 shows the Dice similarity coefficients computed in order to compare the overlap of the left ventricular automatic segmentation and the manual segmentation. As before, the comparisons were made at end-systole and end-diastole.

\section{Discussion}

We developed a method to automatically generate fourdimensional segmentations of the cardiac chambers from 4D Flow MRI data based on a multi-atlas segmentation technique. The resulting segmentations include the entire thoracic cardiovascular system present in the 4D Flow 

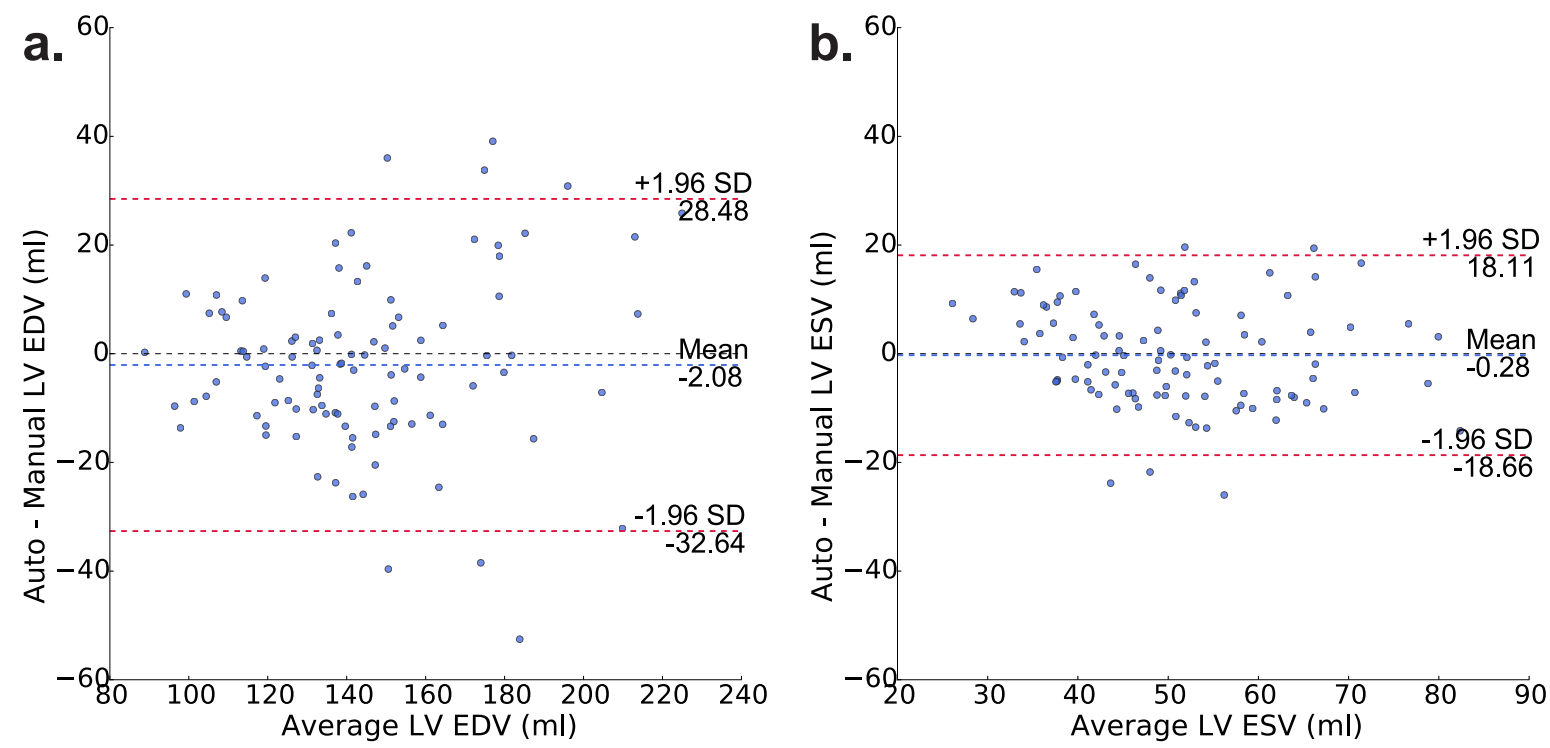

Figure 6: Bland-Altman plots of the volumes obtained for the left ventricle (LV) at end-systole and end-diastole. The values obtained automatically were compared to the ground truth calculated from manually segmented b-SSFP MR images. (a) End-diastolic volume (EDV), (b) end-systolic volume (ESV). The dashed blue line denotes the mean difference. The dashed red lines denote the $95 \%$ limits of agreement $( \pm 1.96 *$ standard deviation $)$

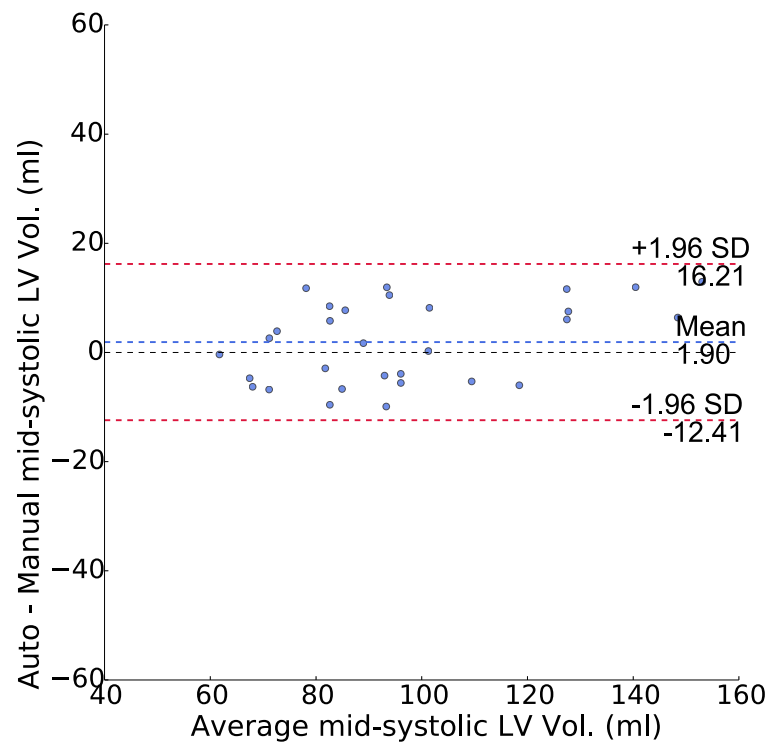

Figure 7: Bland-Altman plots of the volumes obtained for the left ventricle $(\mathrm{LV})$ at a mid-systolic timeframe for a subset of 30 datasets. The values obtained automatically were compared to the ground truth calculated from manually segmented b-SSFP MR images. The dashed red lines denote the $95 \%$ limits of agreement $( \pm 1.96 *$ standard deviation).

MRI data. Evaluation on 102 4D Flow MR datasets from healthy volunteers and patients showed good agreement between manual and automatic segmentations in all the regions assessed.

When comparing the generated volumes with the manually segmented ones, the great majority of the results were within limits of agreement of about $30 \mathrm{ml}$ while most of the average differences between the measurements are between
0 and $3 \mathrm{ml}$, which indicate good agreement between the segmentations. These estimates are comparable to the results from previously reported automatic segmentation methods for b-SSFP MR images (Jolly et al., 2012; Bai et al., 2013, Queiros et al., 2014; Shahzad et al., 2017). Slightly higher values were observed in the left atrium, where the mean differences were between 2 and $7 \mathrm{ml}$. This overestimation could be a consequence of the way the left atrial volumes were estimated in the manual approach through volume integration of long-axis delineations. Cardiac MR segmentations of the left ventricle, obtained from long-axis images, have been found to have a systematic underestimation when compared to the segmentations obtained from short-axis images (Huttin et al. 2015). A more in-depth analysis of the observed errors between the automatic and the manual volumes can be seen in figure 11 . The majority of the errors lie in the zero to ten percent bracket, followed by the ten to twenty percent bracket. As expected, the volumes measured at end-systole in the left ventricle (Fig. $11 \mathrm{~b}$ ), and at end-diastole in the left atrium (Fig. 11 c) are more susceptible to segmentation errors because of their smaller size. Additionally, the left ventricular volume is more difficult to assess during end-systole due to the merging of trabeculae in the myocardial wall. Figure 11 also highlights a few instances in which the error relative to the volume of the segmentation was larger than $40 \%$. For the LV (Fig. 11b), we found that for these cases, the automatic segmentation was typically wider at the cardiac apex and included a larger portion of the papillary muscles when compared to the manual segmentation. However, none of these automatic segmentations where found to be outside the left ventricular area and would still be suitable for flow visualization and assessment. For the left atrium (Fig. 

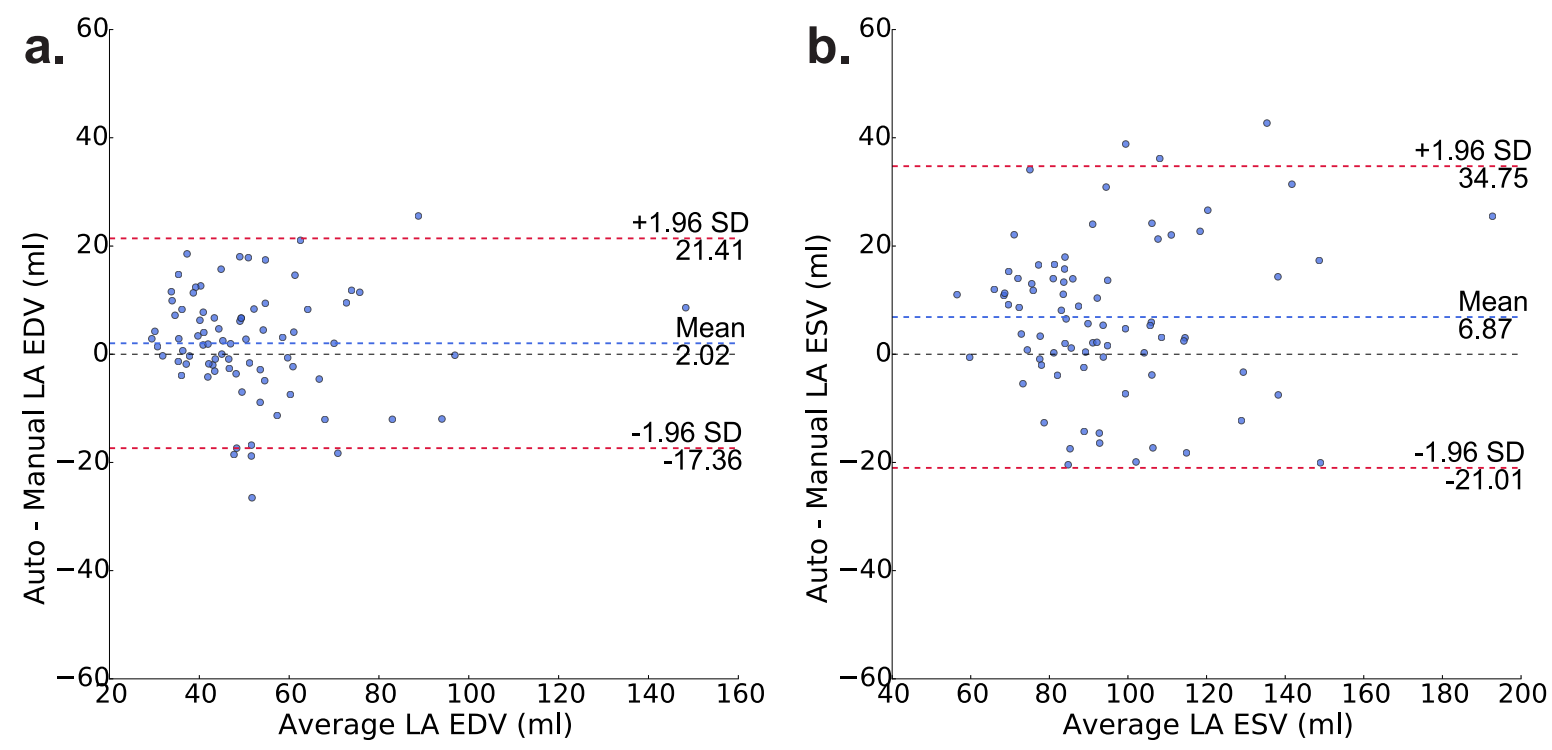

Figure 8: Bland-Altman plots of the volumes obtained for the left atrium (LA). The values obtained automatically were compared to the ground truth calculated from manually segmented b-SSFP MR images. The values compared are: end-diastolic volume (EDV, a), and end-systolic volume (ESV, b). The dashed blue line denotes the mean difference. The dashed red lines denote the $95 \%$ limits of agreement $( \pm 1.96 *$ standard deviation $)$.

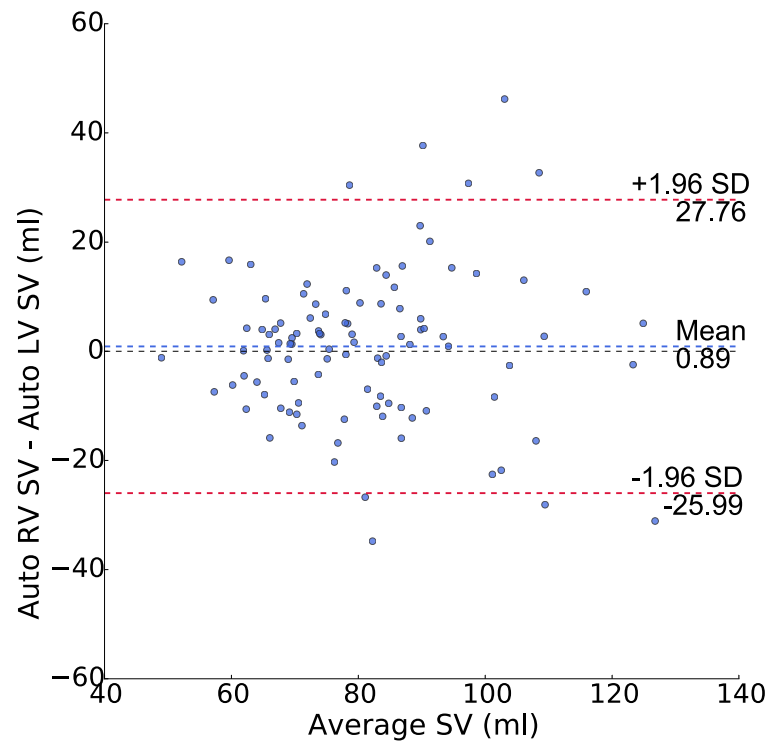

Figure 9: Bland-Altman plots of the stroke volumes obtained in the right ventricle (RV) compared to the left ventricle (LV). The dashed blue line denotes the mean difference. The dashed red lines denote the $95 \%$ limits of agreement $( \pm 1.96 *$ standard deviation $)$.

11 c-d), visual comparison of the two segmentations superimposed on the same image was unfortunately not possible, since the manual values were calculated by integration of a few segmentations performed on long-axis images. However, visual evaluation of the automatic segmentations on the cases were the error relative to the volume was larger than $40 \%$ didn't reveal any obvious failures. The right atrial segmentations were calculated, but not evaluated. This was due to the fact that this cardiac chamber was frequently not fully included in the 4D Flow MR images.
Comparison of ventricular stroke volumes between each other and to the flow volumes estimated in the aorta and pulmonary artery also resulted in good agreement (Fig. 10). This outcome further demonstrates the viability of the automatic segmentations generated by the proposed method.

The segmentation technique was evaluated through volume and similarity comparisons. Unlike the evaluation through volume comparison, the Dice similarity coefficient might be additionally affected by the location of the bSSFP MR SA stack, which can be influenced by the subject's motion during the acquisition of these images (Scott et al. 2009). This is especially noticeable in Fig. 12 where the values reported are, as expected, lower than state-ofthe-art cardiac segmentation methods. Moreover, visual inspection of the most extreme outliers showed that these correspond to datasets with very large differences in the spatial location of the left ventricle on the two MR images compared (b-SSFP MR and 4D Flow MR).

Using the Dice similarity coefficient to measure spatial overlap is a technique frequently used when evaluating automatic segmentation methods. In the present case, however, the automatic and manual segmentations are performed in images from different MR acquisitions (4D Flow MRI and b-SSFP MRI). Therefore, the evaluation outcomes can be affected by the inherent differences between the acquisition methods. For this same reason, surfaceto-surface distances between the automatic and manual contours would not be a reliable measure of comparison in this case. As mentioned previously, b-SSFP MR images typically suffer from misalignments caused by breathing related motion. The automatic correction method applied on the images improved the spatial correspondence between 

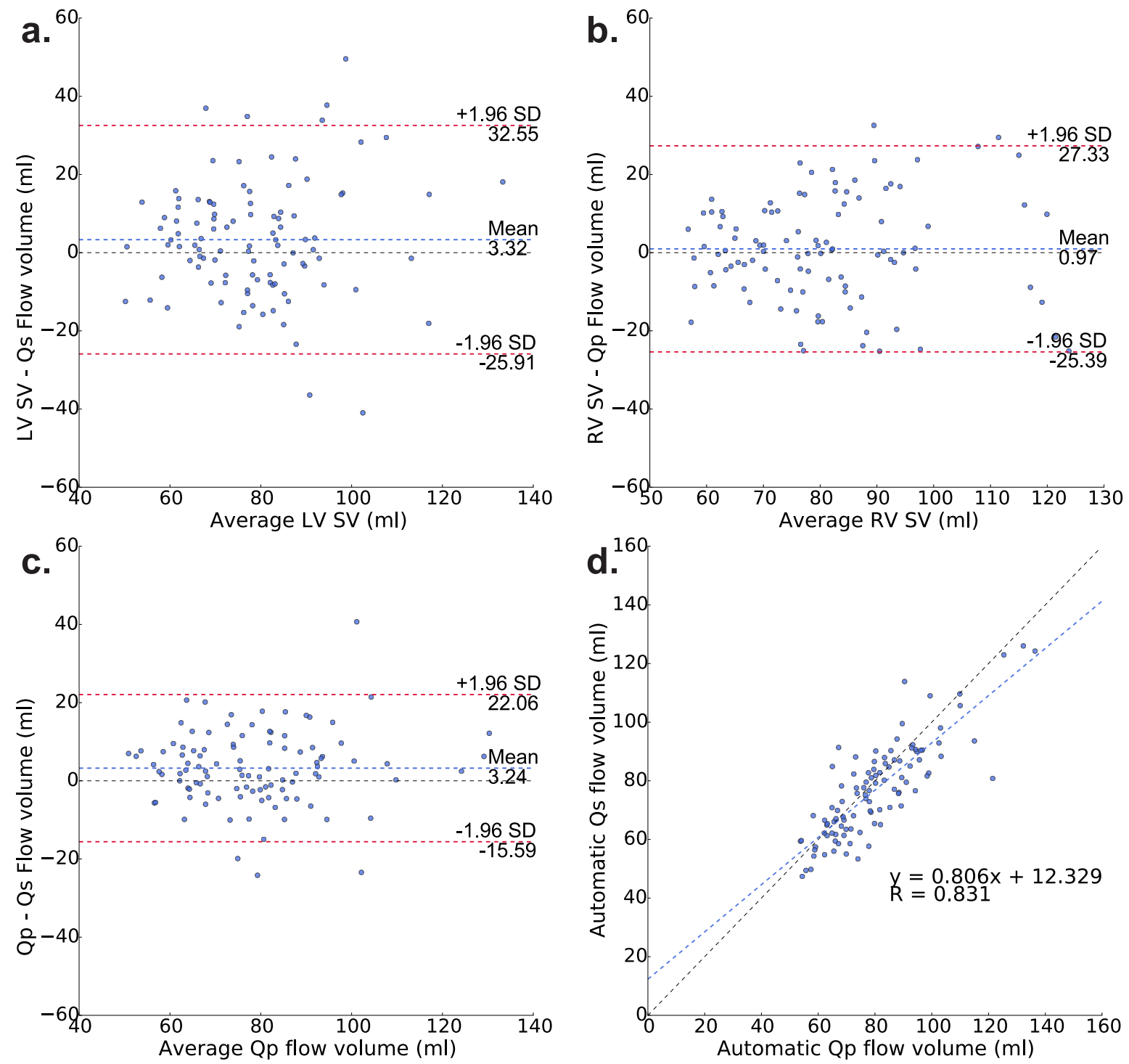

Figure 10: a. Bland-Altman plot comparing the stroke volumes (SV) obtained in the left ventricle (LV) to the flow volumes obtained on planes located in the ascending aorta (systemic flow, Qs). b. Bland-Altman plot comparing the stroke volumes obtained in the right ventricle (RV) to the flow volumes obtained on planes located in the pulmonary artery trunk (pulmonary flow, Qp). In figures c and d, the flow volumes at the Qs and Qp planes are compared using Bland-Altman and correlation analysis. In the Bland-Altman plots, the dashed blue line denotes the mean difference, while the dashed red lines denote the $95 \%$ limits of agreement $( \pm 1.96 *$ standard deviation). In the correlation plot, the regression line is shown in blue. 
a. 69 LV - EDV error relative to volume

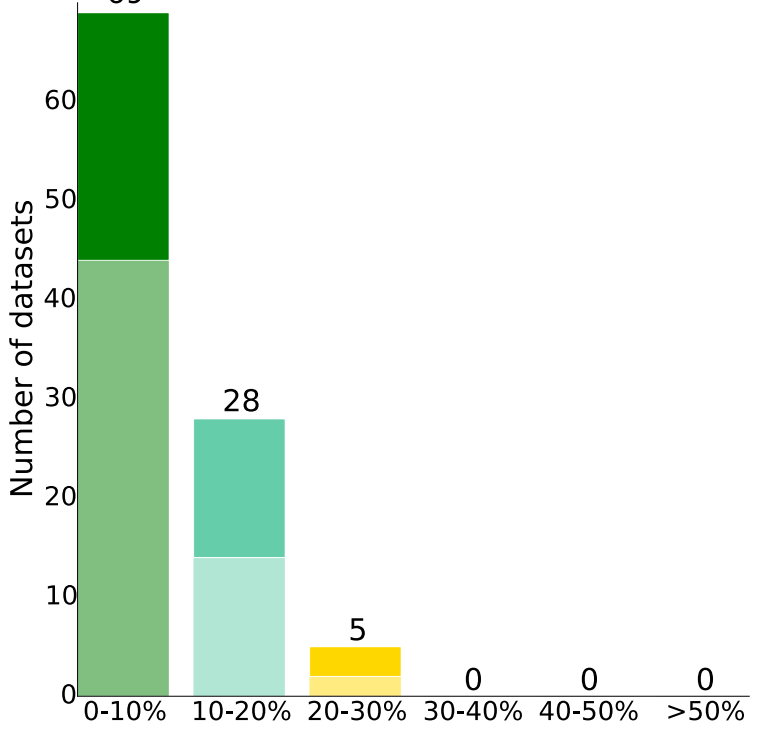

C. 34 LA - EDV error relative to volume

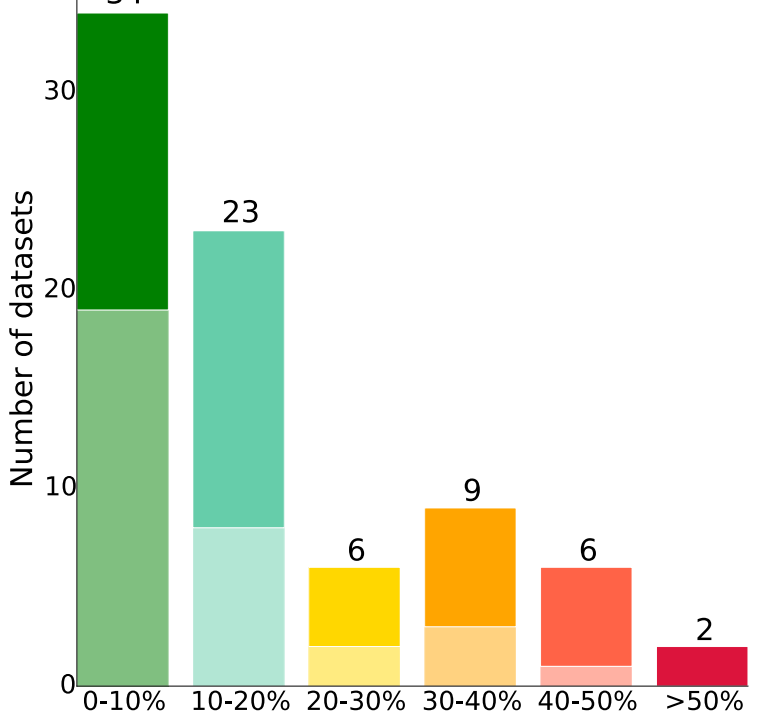

b. LV - ESV error relative to volume

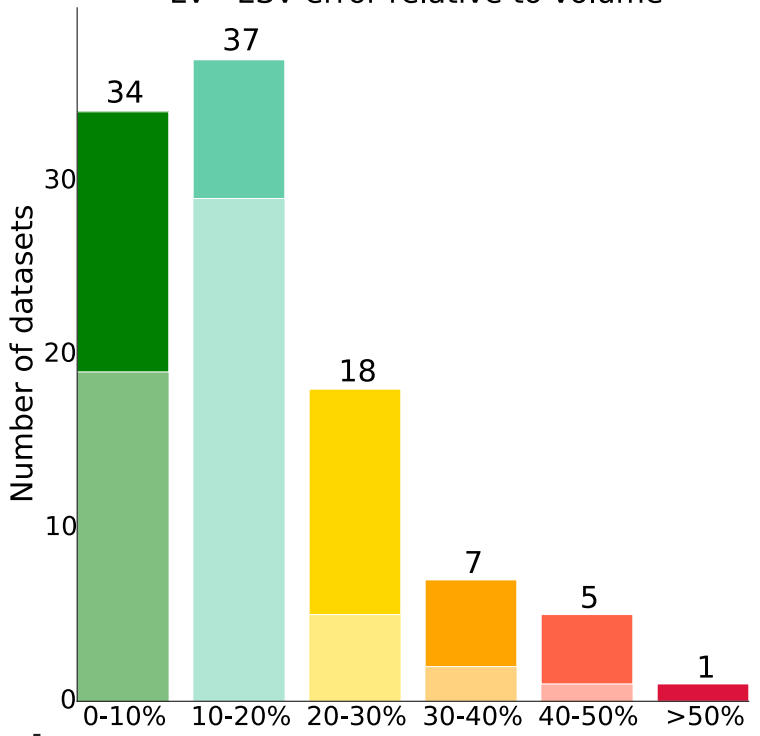

d.

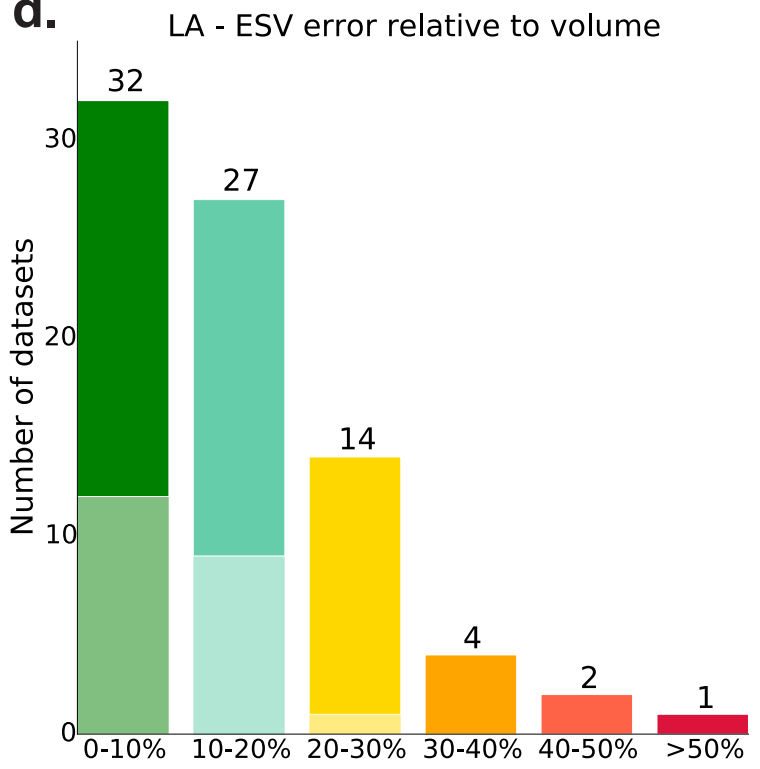

Figure 11: Error relative to volume for the left ventricle (LV, a,b) and left atrium (LA, c,d) at end-diastole (a, c), and end-systole (b, d). The upper section of each bar, in darker color, represents an overestimation of the volume, while the lower sections, in lighter colors, represent an underestimation of the volume. 


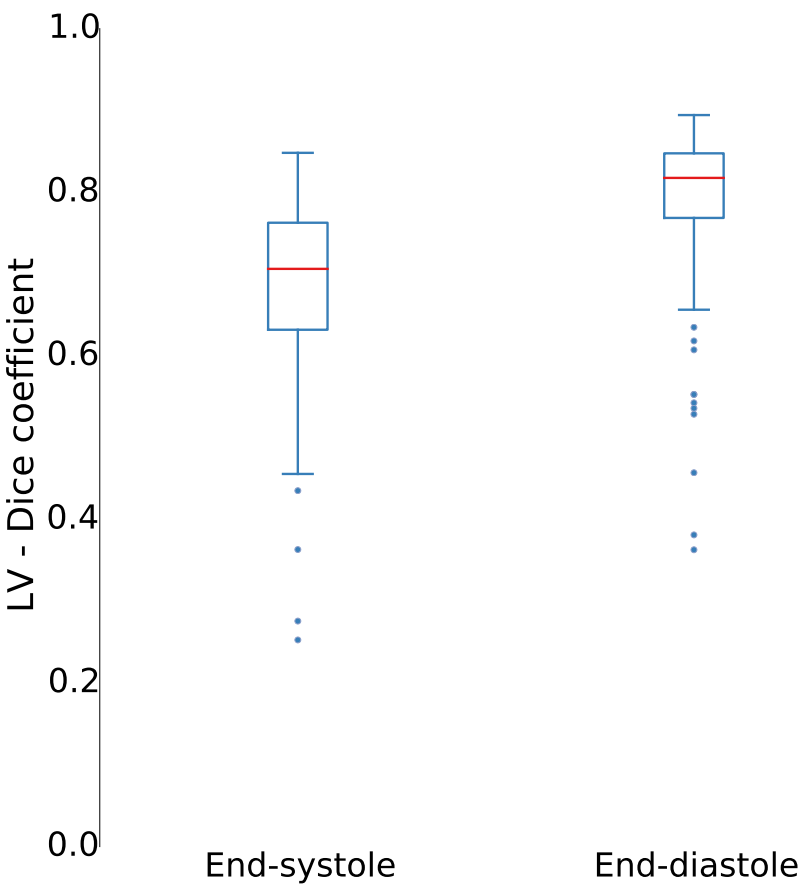

Figure 12: Dice similarity coefficients comparing the automatic and the manual left ventricular segmentations at end-systole (left) and end-diastole (right). The value of the coefficient varies between 0 and 1, where 1 means complete overlap. The red line inside each box indicates the median. The lower and upper edges of the box report first and third quartiles, respectively, while the whiskers reflect confidence intervals.

most acquisitions; however, extreme cases of misplacement remained largely unchanged since they would require a correction method specifically developed for this issue.

The proposed technique uses 4D PC-MRCA data in order to register 4D Flow MRI datasets to each other. Another popular technique for the creation of angiographic images from 4D Flow MRI is 3D PC-MRA, which focuses especially on the great thoracic vessels (Bock et al., 2008). However, slower blood-flows often present inside the heart, such as in the left and right ventricular apex, and left and right atria, are typically not clearly visible in the PC-MRAs. Furthermore, changes in the heart's shape and size over the cardiac cycle are lost during the generation process of these type of angiographies, since they are usually calculated as an average or a maximum intensity projection of all the available timeframes in the $4 \mathrm{D}$ Flow MRI. The 4D PCMRCA data generated from 4D Flow MRI handles these issues by improving blood flow visualization in areas of low velocities, and allowing for comparison of the $4 \mathrm{D}$ Flow MR images at any timeframe (Bustamante et al., 2017).

A common characteristic of the set of datasets included in this study was their ejection fraction (EF), which was chosen to be over $55 \%$. The normal range for this parameter has been found to be $67 \pm 5 \%$ for both men and women of 20 to 80 years of age (Kawel-Boehm et al. 2015). Consequently, even though the included patients suffer from a certain level of heart disease, most of the datasets con- sidered do not present a severely impaired left ventricular function. The atlases generated for this study were also created using datasets acquired from subjects with normal left ventricular function, consequently, the technique es expected to work best on subjects where the ventricular function is not severely impaired. To apply the method on patients with more than moderate left-ventricular systolic dysfunction, we should anticipate a more limited range of motion between end-diastole and end-systole that is not modelled by our atlases. Future work that could be done to adapt the method to a wider degree of cardiac disease includes: using atlases including a single timeframe at middiastole or end-diastole, or creating new atlases from a group of patients with similar levels of dysfunction, which could be used on their own or in addition to the atlases that we have already included. Similarly, for patients with more severe cardiac remodeling, specific sets of atlases that represent the expected cardiac morphology can be included. Prior knowledge about the patient's condition could be used to select the most appropriate set of atlases for a specific subject.

Due to the arduousness and complexity of the manual atlas generation process, only two researchers participated in their creation. A possible improvement to the method could include a larger number of experts during this step in order to guarantee a higher degree of consistency in the atlases used. Moreover, it is also important to work with a set of atlases sufficiently large and varied in order to successfully represent the sizes and shapes of all the hearts to be segmented. The selected set of atlases chosen for this study were able to yield good agreement when comparing the automatic and manual segmentations; however, further work might be necessary to determine the specific size and variability of the atlases necessary to generate satisfactory results within different groups of datasets. Despite the ensuing increase in computation times expected after the inclusion of more atlases, the developed tool would continue to be completely automatic, and calculations would still be performed offline. Adding more atlases to the implementation could also open the possibility of selecting only the more relevant atlases for the target dataset in question. An atlas selection step can improve computational times and assist in the exclusion of atlases that might influence the result in a negative way (Aljabar et al. 2009).

The registration step of the multi-atlas segmentation process was performed at two timeframes: end-systole and end-diastole. Due to the way in which PC-MRCA images are created, they are not affected by the differences in blood flow velocities between the available timeframes of the 4D Flow MRI, and therefore any timeframe could be used for the registration. Initial tests of the developed tool used only one mid-diastolic timeframe during this step, subsequently applying registration between the available timeframes in order to generate a segmentation covering the entire cardiac cycle. When following this procedure, however, the rapid motion of the myocardium and papillary muscles during systole caused an overestimation of 
the end-systolic volumes in the final segmentation. To minimize this problem, both end-systolic and end-diastolic atlases were included in the final method in order to depict the volume changes more accurately. As the datasets used during this study belong to healthy volunteers and patients with cardiac cycles that did not differ significantly from the norm, end-systole and end-diastole presented themselves at similar timeframes in all the evaluated datasets. More research might be necessary to investigate if this approach is sufficient in patients with severely abnormal cardiac cycles; for instance, patients with conduction abnormalities.

In this study, multi-atlas segmentation has been implemented in a relatively simple and straightforward manner. More advanced approaches like label propagation, atlas selection, and label fusion techniques might be able to improve the results further. Also, previously developed extensions to the STAPLE algorithm, allowing it to take into account the shape of the segmentation or regional similarities might be advantageous for this application (Weisenfeld and Warfield, 2011; Asman and Landman, 2013).

The 4D Flow MRI datasets used were obtained after a late-enhancement study was performed on the patients, which required injection of a non-blood pool Gadolinium contrast agent. Consequently, some of the remaining contrast medium was still present in the blood when the $4 \mathrm{D}$ Flow MR images were acquired. The contrast agent available in the images mainly improves the visibility of the myocardium in the magnitude images; therefore, it is most important during the time-resolving steps of the proposed method. Feasibility of the proposed technique on noncontrasted images was not evaluated; although the utilization of non-blood pool and blood pool contrast agents prior to the acquisition of $4 \mathrm{D}$ Flow MRI data is fairly common (Dyverfeldt et al., 2015, Vasanawala et al., 2015).

The major disadvantage of using multi-atlas segmentation is its necessity for computational resources. However, 4D Flow MRI images have a relatively low resolution when compared to other medical imaging techniques, resulting in reasonable computational times (approximately 25 minutes per dataset). The results presented in this study were obtained using parallel computing on a $3.5 \mathrm{GHz}, 6$-core CPU with 64GB RAM. Further developments using specialized computational hardware, such as GPU computing, are expected to result in significantly reduced computational times. This is specially true for the registration technique used, which is also the main consumer of resources throughout the method (Forsberg et al., 2011).

Manual segmentation in MRI is a painstaking process, often associated with rather large intra-observer and interobserver variability. For the left ventricle, Matheijssen et al. (Matheijssen et al., 1996) found intra-observer and interobserver variability to be $3.5 \%$ and $5.2 \%$ for left ventricular endocardial volume, $2.0 \%$ and $2.5 \%$ for epicardial volume, and $3.6 \%$ and $3.6 \%$ for left ventricular mass, respectively. Automation of the segmentation process offers some improvements to manual segmentation on large scale data by making the process faster, more reliable, repeatable, and scalable. Moreover, automatic segmentation methods could be particularly valuable in cases where reproducibility is critical, such as when segmenting follow-up images of the same patient after a procedure or treatment. In the case of 4D Flow MRI, manual and automatic segmentation of the cardiac chambers is even more challenging. This is often circumvented by using segmentations from a separately acquired stack of b-SSFP MR, the result of which is then superimposed on the 4D Flow MRI. Typically in combination with some manual or (semi-)automatic alignment of these datasets (Gupta et al. 2017). Direct segmentation of the 4D Flow MRI data would reduce the length of the acquisition protocol and analysis time. Cine SA MRI images of the heart are acquired for two main reasons: assessment of myocardial status (morphology and motion), and cardiac volume calculation. With the constant improvements in MRI acquisition techniques, 4D Flow MRI could also be used for volume calculation in the future. Currently, however, the generated segmentations suffer from a few limitations due to the inherent characteristics of the $4 \mathrm{D}$ Flow MRI data used, among them, their lower resolution when compared to b-SSFP MR images, which affects the sharpness of the myocardium and can make the visualization of small structures, such as the papillary muscles, very difficult.

For evaluation purposes, this study focused on the segmentation of the heart chambers and major vessels. However, the proposed method may also allow for the segmentation of any other structure within the 4D Flow MR image; for example, the systemic or pulmonary circulation, the left atrial appendage, and the caval veins. This, however, has not been tested in this study.

The proposed method results in segmentations suitable for volume calculation and assessment of hemodynamic markers in the cardiac chambers using 4D Flow MRI. Moreover, the segmentations offer the possibility to visualize and analyze the flow of blood in the cardiovascular system throughout the cardiac cycle, one of the main goals of the 4D Flow MRI acquisition. Blood flow analysis can be performed independently in each region included within the segmentation, or by following the flow of blood in a group of regions. An example can be seen in Fig. 13, where the pulmonary and systemic flows are illustrated at different timeframes of the cardiac cycle.

\section{Conclusion}

Multi-atlas segmentation of 4D Flow MRI datasets permits automated and accurate segmentation of the cardiac ventricles and the large thoracic vessels using only $4 \mathrm{D}$ Flow MR images. The resulting segmentations are fourdimensional and can facilitate flow assessment in the entire thoracic cardiovascular system. 

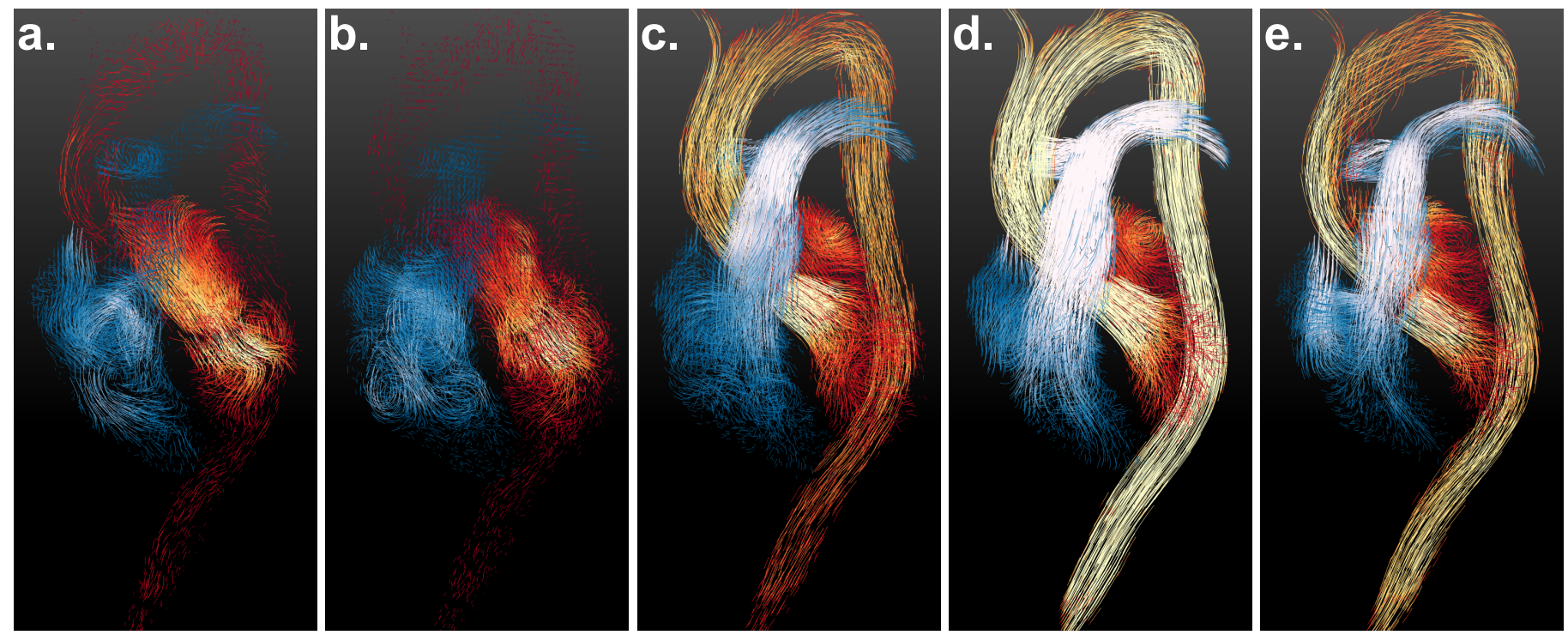

Figure 13: Flow visualization using the segmentations generated by the proposed method at the following timeframes of the cardiac cycle: a. Filling, b. Late filling, c. Early systole, d. Mid-systole, e. End-systole. The pulmonary flows are depicted using a cold palette, and the systemic flows using a warm palette.

\section{Appendix A. The local structure tensor}

The definition of local structure tensor was derived due to the necessity of finding a suitable representation for three-dimensional surface orientation. A local structure tensor $T$ can be calculated by linear summation of the quadrature filter output magnitudes:

$$
T=\sum_{k}\left\|q_{k}\right\|\left(n_{k} n_{k}^{T}-\alpha I\right)
$$

where $\left\|q_{k}\right\|$ is the magnitude of the quadrature filter $k, n_{k}$ is the orientation of the quadrature filter $k, \alpha=\frac{1}{m-1}, m$ being the dimensionality of $T$, and $I$ is the identity tensor.

A quadrature filter can be defined in Fourier space as:

$$
q_{k}= \begin{cases}F_{k}(u)>0, & \text { if } u \cdot n_{k}>0 \\ F_{k}(u)=0, & \text { otherwise }\end{cases}
$$

where $n_{k}$ is the filter directing vector, and $u$ is the frequency. The magnitude $\left\|q_{k}\right\|$ of $q_{k}$ will be phase invariant, and the $\operatorname{argument} \arg \left(q_{k}\right)$ represents the local phase. The minimum number of filters is 3 for two-dimensional space, and 6 for three-dimensional space.

For more details and properties of the local structure tensor please refer to (Knutsson, 1989).

\section{Appendix B. Approximation of left atrial volumes}

Left atrial volumes were approximated by adding the area in each manually segmented long-axis slice using the visualization software Segment (Medviso, Lund, Sweden) (Heiberg et al. 2010). The volume contribution of each slice was given by:

$$
\delta V=\frac{\pi}{2 * Z} \int y(s)^{2} \operatorname{sign}(y(s)) \frac{d x}{d s} d s
$$

where $(x(s), y(s))$ is the parametric representation of the curve, and $Z$ is the number of slices available.

\section{Acknowledgments}

This work was partially funded by the FP7-funded project DOPPLER-CIP [grant number 223615]; the European Union's Seventh Framework Programme (FP7/20072013) [grant number 310612]; the Swedish Research Council [grant number 621-2014-6191]; and the Swedish Heart and Lung Foundation [grant number 20140398].

The authors have no relevant conflicts of interest to disclose.

\section{References}

Aljabar, P., Heckemann, R.A., Hammers, A., Hajnal, J.V., Rueckert, D., 2009. Multi-atlas based segmentation of brain images: Atlas selection and its effect on accuracy. NeuroImage 46, 726-738.

Arvidsson, P.M., Kovács, S.J., Töger, J., Borgquist, R., Heiberg, E., Carlsson, M., Arheden, H., 2016. Vortex ring behavior provides the epigenetic blueprint for the human heart. Scientific Reports 6, 22021.

Asman, A.J., Landman, B.A., 2013. Non-local statistical label fusion for multi-atlas segmentation. Medical Image Analysis 17, 194-208.

Bai, W., Shi, W., O'Regan, D.P., Tong, T., Wang, H., Jamil-Copley, S., Peters, N.S., Rueckert, D., 2013. A probabilistic patch-based label fusion model for multi-atlas segmentation with registration refinement: Application to cardiac MR images. IEEE Transactions on Medical Imaging 32, 1302-1315.

Bajcsy, R., Broit, C., 1982. Matching of deformed images, in: Sixth International Conference on Pattern Recognition (ICPR82), pp. 351-353.

Bieri, O., Scheffler, K., 2013. Fundamentals of balanced steady state free precession MRI.

Bock, J., Wieben, O., Johnson, K., Hennig, J., Markl, M., 2008. Optimal processing to derive static PC-MRA from time-resolved 3D PC-MRI data, in: Proceedings 16th Scientific Meeting, International Society for Magnetic Resonance in Medicine, p. 3053. 
Bustamante, M., Gupta, V., Carlhäll, C.J., Ebbers, T., 2017. Improving visualization of $4 \mathrm{D}$ flow cardiovascular magnetic resonance with four-dimensional angiographic data: generation of a $4 \mathrm{D}$ phasecontrast magnetic resonance CardioAngiography (4D PC-MRCA). Journal of Cardiovascular Magnetic Resonance 19, 47.

Bustamante, M., Petersson, S., Eriksson, J., Alehagen, U., Dyverfeldt, P., Carlhäll, C.J., Ebbers, T., 2015. Atlas-based analysis of 4D flow CMR: Automated vessel segmentation and flow quantification. Journal of Cardiovascular Magnetic Resonance 17, 87.

Christensen, G.E., Rabbitt, R.D., Miller, M.I., 1996. Deformable templates using large deformation kinematics. IEEE Transactions on Image Processing 5, 1435-1447.

Dempster, A.P., Laird, N.M., Rubin, D.B., 1977. Maximum Likelihood from Incomplete Data via the EM Algorithm. Journal of the Royal Statistical Society. Series B 39, 1-38.

Doan, N.T., Orban de Xivry, J., Macq, B., 2010. Effect of intersubject variation on the accuracy of atlas-based segmentation applied to human brain structures, pp. 76231S-76231S-10.

Dyverfeldt, P., Bissell, M., Barker, A.J., Bolger, A.F., Carlhäll, C.J., Ebbers, T., Francios, C.J., Frydrychowicz, A., Geiger, J., Giese, D., Hope, M.D., Kilner, P.J., Kozerke, S., Myerson, S., Neubauer, S., Wieben, O., Markl, M., 2015. 4D flow cardiovascular magnetic resonance consensus statement. Journal of cardiovascular magnetic resonance : official journal of the Society for Cardiovascular Magnetic Resonance 17, 72.

Ebbers, T., Haraldsson, H., Dyverfeldt, P., 2008. Higher order weighted least-squares phase offset correction for improved accuracy in phase-contrast MRI. Proceedings of the International Society of Magnetic Resonance in Medicine 16 39, 1367.

Eklund, A., Forsberg, D., Andersson, M., Knutsson, H., 2011. Using the local phase of the magnitude of the local structure tensor for image registration, in: Lecture Notes in Computer Science (including subseries Lecture Notes in Artificial Intelligence and Lecture Notes in Bioinformatics), pp. 414-423.

Eriksson, J., Carlhall, C., Dyverfeldt, P., Engvall, J., Bolger, A., Ebbers, T., 2010. Semi-automatic quantification of 4D left ventricular blood flow. Journal of Cardiovascular Magnetic Resonance 12,9 .

Fleet, D.J., Jepson, A.D., 1990. Computation of component image velocity from local phase information. International Journal of Computer Vision 5, 77-104. doi 10.1007/BF00056772

Forsberg, D., 2013. Robust Image Registration for Improved Clinical Efficiency: Using Local Structure Analysis and Model-Based Processing. Ph.D. thesis. Linköping University.

Forsberg, D., 2015. Atlas-based registration for accurate segmentation of thoracic and lumbar vertebrae in ct data, in: Recent Advances in Computational Methods and Clinical Applications for Spine Imaging. Springer International Publishing. volume 20, pp. $49-59$.

Forsberg, D., Eklund, A., Andersson, M., Knutsson, H., 2011. PhaseBased Non-Rigid 3D Image Registration: From Minutes to Seconds Using CUDA. Lect. Notes Comp. Sc. 6688, $414-32$.

Forsberg, D., Lundström, C., Andersson, M., Knutsson, H., 2014. Model-based registration for assessment of spinal deformities in idiopathic scoliosis. Physics in Medicine and Biology 59, 311-326.

Fredriksson, A.G., Svalbring, E., Eriksson, J., Dyverfeldt, P., Alehagen, U., Engvall, J., Ebbers, T., Carlhäll, C.J., 2016. 4D flow MRI can detect subtle right ventricular dysfunction in primary left ventricular disease. Journal of Magnetic Resonance Imaging $43,558-565$.

Gupta, V., Bustamante, M., Fredriksson, A., Carlhäll, C.J., Ebbers, T., 2017. Improving left ventricular segmentation in fourdimensional flow MRI using intramodality image registration for cardiac blood flow analysis. Magnetic Resonance in Medicine .

Heckemann, R.A., Hajnal, J.V., Aljabar, P., Rueckert, D., Hammers, A., 2006. Automatic anatomical brain MRI segmentation combining label propagation and decision fusion. NeuroImage 33, $115-126$.

Heiberg, E., Sjögren, J., Ugander, M., Carlsson, M., Engblom, H., Arheden, H., 2010. Design and validation of Segment-freely available software for cardiovascular image analysis. BMC medical imaging 10, 1.

Hudsmith, L.E., Petersen, S.E., Francis, J.M., Robson, M.D., Neubauer, S., 2005. Normal human left and right ventricular and left atrial dimensions using steady state free precession magnetic resonance imaging. J Cardiovasc Magn Reson 7.

Huttin, O., Petit, M.A., Bozec, E., Eschalier, R., Juillière, Y., Moulin, F., Lemoine, S., Selton-Suty, C., Sadoul, N., Mandry, D., Beaumont, M., Felblinger, J., Girerd, N., Marie, P.Y., 2015. Assessment of Left Ventricular Ejection Fraction Calculation on Longaxis Views From Cardiac Magnetic Resonance Imaging in Patients With Acute Myocardial Infarction. Medicine 94, e1856.

Iglesias, J.E., Sabuncu, M.R., 2015. Multi-atlas segmentation of biomedical images: A survey. Medical Image Analysis 24, 205219.

Jolly, M.P., Guetter, C., Lu, X., Xue, H., Guehring, J., 2012. Automatic Segmentation of the Myocardium in Cine MR Images Using Deformable Registration BT - Statistical Atlases and Computational Models of the Heart. Imaging and Modelling Challenges: Second International Workshop, STACOM 2011, Held in Conjunction with MICCAI 2011, Toronto, ON, Canada, September 22, 2011, Revised Selected Papers, Springer Berlin Heidelberg, Berlin, Heidelberg, pp. 98-108.

Kanski, M., Arvidsson, P.M., Töger, J., Borgquist, R., Heiberg, E., Carlsson, M., Arheden, H., 2015. Left ventricular fluid kinetic energy time curves in heart failure from cardiovascular magnetic resonance 4D flow data. Journal of Cardiovascular Magnetic Resonance $17,111$.

Kawel-Boehm, N., Maceira, A., Valsangiacomo-Buechel, E.R., VogelClaussen, J., Turkbey, E.B., Williams, R., Plein, S., et al., 2015. Normal values for cardiovascular magnetic resonance in adults and children. Journal of Cardiovascular Magnetic Resonance 17, 29.

Klein, A., Mensh, B., Ghosh, S., Tourville, J., Hirsch, J., 2005. Mindboggle: automated brain labeling with multiple atlases. BMC medical imaging 5,7 .

Knutsson, H., 1989. Representing Local Structure Using Tensors, in: Proceedings of the 6th Scandinavian Conference on Image Analysis, Linköping University, The Institute of Technology. Linkoöping University Electronic Press. pp. 244-251.

Knutsson, H., Andersson, M., 2005. Morphons: Segmentation using elastic canvas and paint on priors, in: Proceedings - International Conference on Image Processing, ICIP, pp. 1226-1229.

Lang, R.M., Bierig, M., Devereux, R.B., Flachskampf, F.A., Foster, E., Pellikka, P.A., Picard, M.H., Roman, M.J., Seward, J., Shanewise, J.S., Solomon, S.D., Spencer, K.T., St John Sutton, M., Stewart, W.J., 2017. Recommendations for Chamber Quantification: A Report from the American Society of Echocardiography's Guidelines and Standards Committee and the Chamber Quantification Writing Group, Developed in Conjunction with the European Association of Echocardiography, a Branch of the European Society of Cardiology. Journal of the American Society of Echocardiography 18, 1440-1463.

Lantz, J., Henriksson, L., Persson, A., Karlsson, M., Ebbers, T., 2016. Patient-Specific Simulation of Cardiac Blood Flow From High-Resolution Computed Tomography. Journal of biomechanical engineering 138 .

Lorenzo-Valdés, M., Sanchez-Ortiz, G.I., Elkington, A.G., Mohiaddin, R.H., Rueckert, D., 2004. Segmentation of 4D cardiac MR images using a probabilistic atlas and the EM algorithm. Medical Image Analysis 8, 255-265.

Markl, M., Kilner, P.J., Ebbers, T., 2011. Comprehensive 4D velocity mapping of the heart and great vessels by cardiovascular magnetic resonance. Journal of Cardiovascular Magnetic Resonance 13, 7.

Matheijssen, N.a., Baur, L.H., Reiber, J.H., van der Velde, E.a., van Dijkman, P.R., van der Geest, R.J., de Roos, a., van der Wall, E.E., 1996. Assessment of left ventricular volume and mass by cine magnetic resonance imaging in patients with anterior myocardial infarction intra-observer and inter-observer variability on contour detection. International journal of cardiac imaging 12, 11-9.

Petitjean, C., Dacher, J.N., 2011. A review of segmentation methods in short axis cardiac MR images.

Queiros, S., Barbosa, D., Heyde, B., Morais, P., Vilaca, J.L., Fri- 
boulet, D., Bernard, O., D'hooge, J., 2014. Fast automatic myocardial segmentation in $4 \mathrm{D}$ cine $\mathrm{CMR}$ datasets. Medical image analysis 18, 1115-1131.

Rademakers, F., Engvall, J., Edvardsen, T., Monaghan, M., Sicari, R., Nagel, E., Zamorano, J., Ukkonen, H., Ebbers, T., Di Bello, V., Voigt, J.U., Herbots, L., Claus, P., D'hooge, J., 2013. Determining optimal noninvasive parameters for the prediction of left ventricular remodeling in chronic ischemic patients. Scandinavian cardiovascular journal : SCJ 47, 329-34.

Rohlfing, T., Brandt, R., Menzel, R., Maurer, C.R., 2004. Evaluation of atlas selection strategies for atlas-based image segmentation with application to confocal microscopy images of bee brains. NeuroImage 21, 1428-1442.

Rohlfing, T., Brandt, R., Menzel, R., Russakoff, D., Maurer Jr., C., 2005. Quo Vadis, Atlas-Based Segmentation?, in: Suri, J., Wilson, D., Laxminarayan, S. (Eds.), Handbook of Biomedical Image Analysis. Springer US. chapter 11, pp. 435-486.

Rohlfing, T., Russakoff, D.B., Maurer, C.R., 2003. Expectation maximization strategies for multi-atlas multi-label segmentation. Information processing in medical imaging : proceedings of the ... conference 18, 210-21.

Schulz-Menger, J., Bluemke, D.A., Bremerich, J., Flamm, S.D., Fogel, M.A., Friedrich, M.G., Kim, R.J., et al., 2013. Standardized image interpretation and post processing in cardiovascular magnetic resonance: Society for Cardiovascular Magnetic Resonance (SCMR) Board of Trustees Task Force on Standardized Post Processing. Journal of Cardiovascular Magnetic Resonance 15, 35.

Scott, A.D., Keegan, J., Firmin, D.N., 2009. Motion in cardiovascular MR imaging. Radiology 250, 331-351.

Shahzad, R., Tao, Q., Dzyubachyk, O., Staring, M., Lelieveldt, B.P.F., van der Geest, R.J., 2017. Fully-automatic left ventricular segmentation from long-axis cardiac cine MR scans. Medical Image Analysis 39, 44-55.

Suinesiaputra, A., Bluemke, D.A., Cowan, B.R., Friedrich, M.G., Kramer, C.M., Kwong, R., Plein, S., et al., 2015. Quantification of $\mathrm{LV}$ function and mass by cardiovascular magnetic resonance: multi-center variability and consensus contours. Journal of Cardiovascular Magnetic Resonance 17, 63.

Suinesiaputra, A., Cowan, B.R., Al-Agamy, A.O., Elattar, M.A., Ayache, N., Fahmy, A.S., Khalifa, A.M., Medrano-Gracia, P., Jolly, M.P., Kadish, A.H., Lee, D.C., Margeta, J., Warfield, S.K., Young, A.A., 2014. A collaborative resource to build consensus for automated left ventricular segmentation of cardiac MR images. Medical Image Analysis 18, 50-62.

Vasanawala, S.S., Hanneman, K., Alley, M.T., Hsiao, A., 2015. Congenital heart disease assessment with 4D flow MRI. Journal of magnetic resonance imaging : JMRI 42, 870-86.

Vercauteren, T., Pennec, X., Perchant, A., Ayache, N., 2009. Diffeomorphic demons: Efficient non-parametric image registration. NeuroImage 45, S61-S72.

Warfield, S.K., Zou, K.H., Wells, W.M., 2004. Simultaneous truth and performance level estimation (STAPLE): An algorithm for the validation of image segmentation. IEEE Transactions on Medical Imaging 23, 903-921.

Weisenfeld, N.I., Warfield, S.K., 2011. SoftSTAPLE: Truth and performance-level estimation from probabilistic segmentations.

Xiang, Q.S., 1995. Temporal phase unwrapping for CINE velocity imaging. Journal of magnetic resonance imaging : JMRI 5, 529-34

Yushkevich, P.A., Piven, J., Hazlett, H.C., Smith, R.G., Ho, S., Gee, J.C., Gerig, G., 2006. User-guided 3D active contour segmentation of anatomical structures: Significantly improved efficiency and reliability. NeuroImage 31, 1116-1128.

Zhang, J., Wang, J., Wang, X., Feng, D., 2015. Multimodal image registration with joint structure tensor and local entropy. International journal of computer assisted radiology and surgery 10 , $1765-1775$ 